\title{
Steady flows and unsteady ones in pipes: models link and quasistationarity hypothesis
}

\author{
Vladimir $V$. Tarasevich ${ }^{1, *}$ \\ ${ }^{1}$ NB IWEP SB RAS, 2, Morskoy Ave, 2, Novosibirsk, 630090, Russian Federation
}

\begin{abstract}
Models of unsteady flows and stationary ones in pipes and the requirements of their joining are considered. In this view, the problem of the description of a friction at a non-stationary flow is considered. So-called "quasi-stationarity hypothesis" is discussed. The formula for a friction coefficient generalized on a non-stationary case is offered. It is concluded that additional losses at the non-stationary mode are caused by energy consumptions for reorganization of speed chart.
\end{abstract}

\section{Introduction}

The processes occurring in pipeline systems can be divided into stationary processes and nonstationary ones. The theory of hydraulic circuits [1] considers stationary problems. In the general case, the flow here is described by the Bernoulli equation taking into account pressure losses $[2,12]$. The pressure losses, in turn, may be found using the well-known DarcyWeisbach formula $[2,13]$.

The well-known water hammer problem [4, 9] belongs to category of non-stationary problems, for example. In this case the process parameters are described by a onedimensional system of partial differential equations of hyperbolic type.

To the problems as which are correctly formulated, different requirements of correctness are usually imposed: correctness according to J. Hadamard [5], correctness according to N.A. Tikhonov [6]; the requirements of boundary conditions' correctness are formulated in the book of B.I. Rozhdestvensky and N.N. Yanenko [7].

The requirements, which, in a sense, can also be interpreted as requirements of correctness, should include the requirement of the non-stationary models docking to the corresponding stationary ones.

In the same way as a non-stationary flows can develop from an initial stationary flows, or vice versa, the corresponding mathematical stationary models and non-stationary ones should also transit into each other smoothly.

For example, the equations of water hammer $[4,9]$ in the stationary case (when all time derivatives are equal to zero) should be "transformed" into the Bernoulli equation; the SaintVenant equations [8], describing the water flow in the channel, should be transformed the corresponding equations of steady uneven flow [2], etc.

\footnotetext{
*Corresponding author: tvv@academ.org
} 
The requirement of model joining can serve as additional criterion for the adequacy of the approach used, for example, at justification of a so-called quasi-stationarity hypothesis [10].

This hypothesis assumes that friction losses in case of unsteady flow can be calculated by the dependences for the steady flow.

The fact is that the classical equations of water hammer in pipes, proposed by N.E. Zhukovsky [9], did not contain a term taking the friction losses into account. They gave the equations for the flow of an ideal inviscid fluid while transit to stationary flow model.

It is considered [11] in the Russian scientific literature that the hypothesis of quasisteadiness was firstly proposed by S.A. Khristianovich [12], who suggested that the tension on pipe walls while an unsteady flow depends only on the instantaneous average (by crosssection) velocity, and this dependence has the same form as for the steady flow.

In this case, the considered equations pass into the Bernoulli equation while transition to the models describing stationary flows. Bernoulli's equation takes into account the losses and is used widely for hydraulic calculations of pipelines [2, 13-15].

As it is noted in [10], such an approach gives sufficiently adequate results for low-velocity flows with weak wave processes. The acceptability of the quasi-stationary approach is confirmed by the long-term practice of practical calculations of unsteady flows in pipelines.

However, there are a number of cases when the quasi-stationary model does not give satisfactory results. Thus, in the case of high-intensity hydraulic shocks in short pipelines, a significant smoothing of the fronts of pressure waves is observed, and even a slight decrease in the frequency of oscillations. In the framework of the classical theory of water hammer, this phenomenon is not explained.

Apparently, W. Zielke was the first who paid attention to this circumstance. He proposed the model of "frequency-dependent friction" in his article [19]. For example, attenuation of a sound signal in the air can be an analogy, when higher-frequency harmonics decay faster then low-frequency ones. The Zielke model uses a special weight function that takes into account the "pre-history of the process" and considers the laminar flow.

W. Zielke's paper stimulated the manifestation of interest in this subject and caused emergence of a large number of works. In particular, Zielke's theory was also generalized for the turbulent case in the works of the Russian researcher D.N. Popov [20].

Detailed analysis of various ways to account for the nonstationarity of friction is given in the paper [18]; it also contains the detailed comparative analysis of two theories of transient friction - the aforementioned work of W. Zielke and the theory of B. Brunone et al [21].

\subsection{Disadvantages of approach of W. Zielke}

In a sense, the use of the notion of frequency can be considered as disadvantage of the W.Zielke model. The emergence of "frequencies" is associated with the use of expansion in a Fourier series, which in its physical essence is based on the representation of the oscillatory process as a set of standing waves.

However, during a water hammer there is only one carrier frequency of pressure waves associated with the run of the water hammer wave from the pipe extremity to the other one, and back.

The period of such oscillations will be $T=2 L / c$, and the frequency $v=1 / T$, where $L$ is the pipe length and $c$ is the water hammer wave velosity. The using some additional frequencies seems to be somewhat artificial.

That is why Zielke is limited to laminar flow, because in this case we have linear equations, to which the Fourier series decomposition can be applied formally. 
However, for turbulent flows, the corresponding system of water hammer equations will be non-linear one (more precisely, semi-linear one, in the terminology of [7]), to which the Fourier apparatus is inapplicable.

Let's note also that application of weight function of the "stream prehystory" is equivalent essentially to the use of higher order derivatives.

All aforesaid leads to the idea that the original water hammer model itself has to contain the certain additional members responsible for effects of non-stationary friction.

Then there will be no need for an artificial introduction of frequency and other artificial techniques. Identifying these additional terms requires a more rigorous obtaining the basic equations.

\section{Mathematical formulation of problem}

The flow of a weakly compressible liquid $[2,8,14]$ in a low-elastic pipeline is considered. That is, the dependencies the liquid density $\rho$ and the cross-sectional area $S$ on pressure will be assumed to be small; moreover, if these quantities are involved in the coefficients at the derivatives, they are treated as constant, but the derivatives of them are not small and are taken into account in the equations.

The Cartesian coordinate system is used, with the $x$ axis directed along the pipe (coordinate along the length), and the $y$ and $z$ axes are located in the transverse section plane.

We will operate with quantities averaged over the cross section. Let $\rho=\rho(x, t)$ be the averaged density of the liquid. The mass flow rate $M$ will be understood as the mass of fluid flowing through the pipe cross-section per unit of time; the volume flow rate $Q$ is defined as $Q=M / \rho$, the average flow velocity $V$ is defined as $V=Q / S$.

Here $t$ is time coordinate.

Let consider a part of pipeline that is enclosed between two cross-sections located at a small distance from each other (Fig. 1).

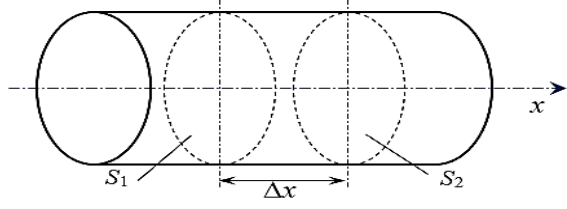

Fig. 1. Pipe section

\subsection{Continuity equation}

Considering the mass balance for the pipe section in Fig. 1, one can obtain the following equation under $\Delta x \rightarrow 0$

$$
\frac{\partial(\rho S)}{\partial t}+\frac{\partial M}{\partial x}=0
$$

The equation (1) transforms into evident equality of constant mass rate along the pipe for steady flow $(\partial(\rho S / \partial t)=0)$

$$
M=\text { const } .
$$

This equality holds true for the flow of any fluid (including gas) in a pipeline with both rigid walls (as steel ones) and pliable walls (as a rubber hose, for example).

If we consider the flow of weakly compressible fluid (water belongs to which, for example) in a pipe with sufficiently rigid walls, then equality (2) can be represented as

$$
V=\text { const, }
$$

i.e. as the requirement of constant velocity in cylindrical pipe.

\subsection{Equation of motion}


The equation of motion is obtained by applying the law of the momentum conservation to the pipe section in Fig.1.

Before starting the obtaining the equation of motion, let consider the question of an impulse stream through the cross section $S$.

\subsubsection{Impulse stream through the cross section}

Impulse stream through the cross section $S$ is defined as

$$
\vec{J}=\int_{S} \rho V_{n}^{*} \cdot \vec{V}^{*} d S .
$$

Where $\dot{V}^{*}=\dot{V}^{*}(t, x, y, z)$ is local velosity vector (i.e. the velosity at each point of the cross-section); $V_{n}^{*}=\vec{V}^{*} \cdot \vec{n}$ is this velosity projection on the normal $\vec{n}$ to the surface $S$.

As the one-dimensional flow is considered, the impulse stream projection to axis $x$ is of the greatest interest. Since the $x$ axis is perpendicular to the cross-sectional plane $S$ by definition, then the velocity projection on the normal to this surface will be nothing more than a component of the local velocity along the $x$ axis. In this case, one can have from formula (4)

$$
J_{x}=\int_{S} \rho V_{x}^{*} \cdot V_{x}^{*} d S
$$

Let us express the projection of the momentum flux $J_{x}$ through average values. Based on the formula (5), we can write

$$
J_{x}=\int_{S} \rho V_{x}^{*} \cdot V_{x}^{*} d S=\int_{S} \rho \cdot\left[V^{2}+(\Delta V)^{2}\right] d S=M \cdot V \cdot\left(1+\varepsilon_{0}\right) .
$$

where $\Delta V$ is the difference between the average speed in the section and the local speed at the point of the section. Here

$$
\varepsilon_{0}=\frac{1}{\rho S \cdot V^{2}} \int_{S} \rho \cdot(\Delta V)^{2} d S=\frac{\int_{S} \rho \cdot(\Delta V)^{2} d S}{M \cdot V} \geq 0
$$

is the relative square deviation of the local velocity from the average velocity.

The parameter

$$
\alpha_{0}=1+\varepsilon_{0}
$$

is called the Boussinesq coefficient [2,3].

The Boussinesq coefficient is affected by the uneven distribution of the velocity profile across the cross section. So, if the velocities are the same throughout the section (the velocity

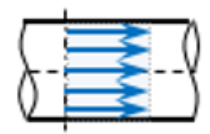

a)

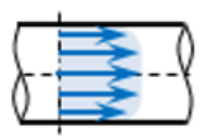

b)

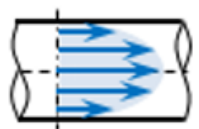

c)

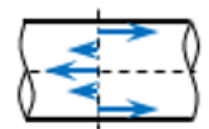

d)

Fig. 2. Velocities' profiles distribution diagram is a rectangle, see Fig. 2, $a$ ), then $\alpha_{0}=1$. For a turbulent flow (Fig. 2, b) $\alpha_{0} \approx 1.03$; and $\alpha_{0}=4 / 3$ for laminar flow (Fig. $2, c)$. However, if the velocity distribution over the cross section is multidirectional, for example, the flow is directed in one direction at the pipe walls, and the flow is directed in the opposite direction in the flow center (Fig. 2, d), then the value $\alpha_{0}$ can be arbitrarily large: $\alpha_{0} \rightarrow \infty$. 


\subsubsection{Equation of motion}

The law of conservation of momentum in relation to the pipe section in Fig. 1 can be formulated as follows

$$
\begin{gathered}
\int_{S} \rho V d S \Delta x\left(t_{2}\right)-\int_{S} \rho V d S \Delta x\left(t_{1}\right)= \\
=\left.\alpha_{01} \rho M V\right|_{1} \Delta t-\left.\alpha_{02} \rho M V\right|_{2} \Delta t+[\oint_{S_{F}}\left(p n_{x}+\tau_{x}\right) d S+g \rho S \underbrace{\Delta x \sin \alpha}_{-\Delta z}] \cdot \Delta t
\end{gathered}
$$

Here is a change of the impulse in the pipe part between cross-sections $S_{1}$ and $S_{2}$ in the left part of the equality, and the total impulse flow through the side faces of the section under consideration and the impulse of all acting forces as surface forces (first term in square brackets) and as mass ones (second term square brackets) are on the right part of (9). Here, $S_{F}$ is the total surface of the pipe section, including the side sections $S_{l}$ and $S_{2}$, and the internal surface of the pipeline; $\alpha$ is the angle of pipeline inclination to the horizon, $\tau_{x}$ is the longitudinal friction stress.

Using the Gauss - Ostrogradsky theorem and passing to the limit under $\Delta x \rightarrow 0$ and $\Delta t \rightarrow 0$, one can obtain from (9)

$$
\frac{\partial V}{\partial t}+V \frac{\partial V}{\partial x}+\frac{1}{\rho S} \frac{\partial\left(\varepsilon_{0} M V\right)}{\partial x}+\frac{1}{\rho} \frac{\partial p}{\partial x}+\frac{1}{\rho S} \frac{\partial}{\partial x} \int_{S} \tau_{x} d S+\frac{\tau_{x} \chi}{\rho S}+g \frac{d z}{d x}=0,
$$

where $\varepsilon_{0}$ is defined in (7), $\chi$ is wetted perimeter.

The shear stress at the pipe walls can be expressed by the well-known hydraulics formula $[2,13-15]$

$$
\tau_{x} \approx \lambda \frac{\rho|V| V}{8}
$$

where $\lambda$ is hydraulic friction coefficient in the Darcy formula [2, $13-15]$.

The term with the friction stress in the cross section can be estimated as

$$
\frac{\partial}{\partial x} \int_{S} \tau_{x} d S \approx \mu S \frac{\partial^{2} V}{\partial x^{2}}
$$

where $\mu$ is the reduced dynamic viscosity.

Substituting these dependencies into formula (10), one can obtain the equation of motion in the form

$$
\frac{\partial V}{\partial t}+V \frac{\partial V}{\partial x}+\frac{1}{\rho} \frac{\partial p}{\partial x}+g \frac{d z}{d x}+\frac{1}{\rho S} \frac{\partial\left(\varepsilon_{0} M V\right)}{\partial x}+\frac{\mu}{\rho} \frac{\partial^{2} V}{\partial x^{2}}+\frac{\lambda|V| V}{8 R_{H}}=0,
$$

where $R_{H}=S / \chi$ is hydraulic radius [2].

Taking into account formula (7) and the weak compressibility of liquid, one can obtain

$$
\frac{\partial V}{\partial t}+V \frac{\partial V}{\partial x}+\frac{1}{\rho} \frac{\partial p}{\partial x}+g \frac{d z}{d x}+\frac{1}{S} \frac{\partial}{\partial x}\left(\int_{S}(\Delta V)^{2} d S\right)+\frac{\mu}{\rho} \frac{\partial^{2} V}{\partial x^{2}}+\frac{\lambda|V| V}{8 R_{H}}=0 .
$$

It follows from the requirement of matching the models that equation (14) in the stationary case has to transform into the well-known Bernoulli equation. Let's check, whether so it. First, local acceleration disappears in the stationary case: $\partial V / \partial t=0$.

Secondly, according to relation (3), the velocity can be considered constant one along the cylindrical tube, therefore, it will be fair 


$$
\frac{\partial^{2} V}{\partial x^{2}}=0
$$

and the integral will be according to (7)

$$
\frac{1}{S} \int_{S}(\Delta V)^{2} d S=\varepsilon_{0} V^{2}
$$

Then, assuming the fluid to be weakly compressible $(\rho \approx$ const $)$, one can obtain from equation (14) and taking into account (8)

$$
\frac{d}{d x}\left(z+\frac{p}{\rho g}+\alpha_{0} \frac{V^{2}}{2 g}\right)+\frac{\lambda|V| V}{8 g R_{H}}=0
$$

This equation is nothing but the Bernoulli equation taking the losses into account $[2,13$ 15]. The only difference is that usually the Bernoulli equation uses so-called correction of kinetic energy (Coriolis coefficient) $\alpha$ at the velocity head. The relationship between the Coriolis and Boussinesq coefficients is given by the well-known formula

$$
\alpha \approx 3 \alpha_{0}-2
$$

The error introduced by this difference is very small. Firstly, the contribution of velocity head is usually small compared to the contribution of hydrostatic head, secondly, the difference between $\alpha_{0} V^{2} / 2 g$ and $\alpha V^{2} / 2 g$ is insignificant, and, thirdly, it remains disputable in general what the coefficient has nevertheless to stand in Bernoulli's equation if it expresses law of momentum conservation?

Thus, the loss of the flow specific energy ("head") in a pipe section with a length $\Delta l$ during unsteady motion in the general case will be given by

$$
\frac{\Delta e}{\Delta l}=\frac{\lambda|V| V}{8 g R_{H}}+\frac{1}{g S} \frac{\partial}{\partial x}\left(\int_{S}(\Delta V)^{2} d S\right)+\frac{\mu}{\rho g} \frac{\partial^{2} V}{\partial x^{2}}
$$

where the first term in the right-hand part of (19) is a "quasi-stationary" loss, and the second term and third ones are associated with the energy loss for restructuring the velocity diagram.

By reducing this energy loss formula to the form of the Darcy-Weisbach formula, it is possible to determine the coefficient of hydraulic friction for the non-stationary case $\lambda_{n s}$ as

$$
\lambda_{n s}=\lambda+\frac{8 R_{H}}{S|V| V}\left[\frac{\partial}{\partial x}\left(\int_{S}(\Delta V)^{2} d S\right)+v \cdot S \frac{\partial^{2} V}{\partial x^{2}}\right]
$$

where $\lambda$ is "usual" coefficient of hydraulic stationary friction, and "additive" to $\lambda$ takes into account non-stationarity.

\section{Conclusion}

The use of the "quasi-stationarity hypothesis", i.e. the use of dependencies that are valid in the case of a stationary flow, to determine the pressure losses in a non-stationary flow, gives a completely acceptable result for the flows with small gradients; but in areas with abrupt fronts of flow parameters, this hypothesis gives a result that deviates strongly from the real situation. 
The presence of such a deviation is associated with the restructuring of the velocity diagram. This restructuring consumes additional energy of the flow, which is perceived as additional losses.

The proposed formulas (19) and (20) are an attempt to consider these additional losses.

A more rigorous approach, according to the author, should be based on a careful averaging of the Navier-Stokes equations $[16,17]$ over the cross section in order to obtain one-dimensional equations for the averaged parameters.

\section{References}

1. A.P. Merenkov, V.Ya. Khasilev, Theory of hydraulic circuits (Nauka Publisher, Moscow, 1985) [in Russian]

2. P.G. Kisilev, Hydraulics: The basic of fluid mechanics (Energia Publisher, Moscow, 1980) [in Russian]

3. A.D. Altshul, Hydraulic Resistancies (Nedra Publisher, Moscow, 1982) [in Russian]

4. A.A. Atavin, V.L. Lavrentiev, V.V. Tarasevich, Hydraulic Circuits. The theory devellopment and applications, 16 (Nauka, Novosibirsk, 2000) [in Russian]

5. J. Hadamard, Sur les problèmes aux dérivées partielles et leur signification physique (1902)

6. M.M. Lavrentiev, V.G. Romanov, S.P. Shishatsky, Incorrect problems of mathematical physics and analysis (Nauka Publisher, Moscow, 1980) [in Russian]

7. B.I. Rozhdestvensky, N.N. Yanenko, Systems of quasi-linear equations and their applications to gas dynamisc (Nauka Publisher, Moscow, 1978) [in Russian]

8. J. J. Stoker, Water waves (Interscience Publishers, Inc., New York, 1957)

9. N.E. Zhukowsky, Bulletin of the Polytechnic Society, 5 (1899) [in Russian]

10. V.E. Seleznev, V.V. Aleshin, S.N. Pryalov, Mathematical Modelling of piping networks and canal systems (Direct Media, Moskow - Berlin, 2014) [in Russian]

11. I.M. Astrakhan, K.S. Basniev, G.I. Barenblatt, G.D. Rosenberg, Professor I.A. Charny (1909-1967) (Neft' i Gas Publisher, Moscow, 1995) [in Russian]

12. S.A. Khristianovich, Unsteady moving in the canals and rivers (Publisher of Academy of Sciencies of USSR, Moscow - Leningrad, 1938) [in Russian]

13. V.L. Streeter, E.B. Wylie, Hydraulic Transients (Mc Graw-Hill, N.Y., 1968)

14. Faith A. Morrison, An Introduction to Fluid Mechanics (Cambridge University Press, New York, 2013)

15. Y. Nakayama, R.F. Boucher, Introduction to Fluid Mechanics (Arnold, GB, 1999)

16. G.K. Batchelor, An Introduction to Fluid Dynamics (Cambridge University Press, New York, 2000)

17. J. Serrin, Mathematical Principles of Classical Fluid Mechanics (Handbuch der Physik, Berlin - Göttingen - Heldelbeg, 1959)

18. A. Bergant, A.R. Simpson, J. Vitkovsky, JHR, 39, 3 (2001)

19. W. Zielke, J. Basic Eng, 90, 1 (1968)

20. D.N. Popov, Unsteady hydro-mechanical processes (Mashinostroenie Publisher, Moscow, 1982) [in Russian]

21. B. Brunone, U.M. Golia, M. Greco, Some remarks on the momentum equation for fast transients, Int. Meeting on Hydraulic Transients with Column Separation, $9^{\text {th }}$ Round Table (IAHR, Valencia, Spain, 1991) 